\title{
Shrinkage Testimators for the Inverse Dispersion of the Inverse Gaussian Distribution under the LINEX Loss Function
}

\author{
Gyan Prakash and D. C. Singh \\ Harishchandra P. G. College, Varanasi, India
}

\begin{abstract}
In this paper, shrinkage testimators for the inverse dispersion for inverse Gaussian distribution when its prior information is available in the form of a guess value have been considered. The proposed testimators have been compared with the minimum risk estimator in the class of unbiased estimators under the LINEX loss function.
\end{abstract}

Zusammenfassung: In dieser Arbeit werden shrinkage testimators für die inverse Dispersion einer inversen Gauss Verteilung betrachtet, falls auch Vorinformation in Form eines vermuteten Wertes vorliegt. Die vorgeschlagenen testimators werden mit dem minimalen Risiko Schätzer in der Klasse der unverzerrten Schätzer unter der LINEX Verlust Funktion verglichen.

Keywords: Shrinkage Factor, Mean Squared Error, Level of Significance.

\section{Introduction}

The inverse Gaussian distribution is used as an important mathematical model for the analysis of positively skewed data. The review article by Folks and Chhikara (1978) and Seshadri (1998) have proposed many interesting properties and applications of this distribution. The probability density function $f(x \mid \mu, \lambda)$ of the inverse Gaussian distribution $\mathrm{IG}(\mu, \lambda)$, is given by

$$
f(x \mid \mu, \lambda)=\sqrt{\frac{\lambda}{2 \pi x^{3}}} \exp \left(-\frac{\lambda(x-\mu)^{2}}{2 \mu^{2} x}\right), \quad x, \mu, \lambda>0 .
$$

Here $\mu$ stands for the mean and $\lambda$ for the inverse measure of dispersion. Let $x_{1}, \ldots, x_{n}$ be a random sample of size $n$ drawn from $\operatorname{IG}(\mu, \lambda)$. The maximum likelihood estimates of $\mu$ and $\lambda$ are

$$
\hat{\mu}=\frac{1}{n} \sum_{i=1}^{n} x_{i}=\bar{x} \quad \text { and } \quad \hat{\lambda}=\frac{n}{v}, \quad \text { where } \quad v=\sum_{i=1}^{n}\left(\frac{1}{x_{i}}-\frac{1}{\bar{x}}\right) .
$$

It is well known that $\bar{x}$ and $\tilde{\lambda}=(n-3) / v$ are unbiased estimates of $\mu$ and $\lambda$, respectively. Also, $\bar{x} \sim \operatorname{IG}(\mu, n \lambda)$ and $\lambda v \sim \chi_{n-1}^{2}$, with $\bar{x}$ and $v$ being stochastically independent (see Tweedie, 1957a, 1957b; Folks and Chhikara, 1978).

Varian (1975) and Zellner (1986) proposed an asymmetric loss function known as the LINEX loss function for any parameter $\lambda$ as

$$
L\left(\Delta^{\prime}\right)=b^{\prime}\left(\exp \left(d \Delta^{\prime}\right)-d \Delta^{\prime}-1\right), \quad d \neq 0, \quad b^{\prime}>0, \quad \text { and } \quad \Delta^{\prime}=\hat{\lambda}-\lambda,
$$


where $\hat{\lambda}$ is an estimate of $\lambda$. We reparameterize the class of the LINEX loss function in the form

$$
L(\Delta)=b(\exp (a \Delta)-a \Delta-1), \quad a \neq 0
$$

with the new scale parameter $b=2 b^{\prime} / a^{2}>0$ and $\Delta=(\hat{\lambda}-\lambda) / \lambda$.

The sign and magnitude of $a$ represents the direction and degree of asymmetry, respectively. A positive (negative) value of $a$ is used when overestimation is more (less) serious than underestimation. For $a$ near to zero, $L(\Delta)$ is approximately square error and therefore it is almost symmetric.

One may have a guess value $\lambda_{0}$ of $\lambda$, which one may like to use to construct a parameter estimate. If this guess value is close to the true value the shrinkage technique is useful to get improved estimators. Following Thompson (1968), shrinkage estimators for the inverse dispersion $\lambda$ are given by

$$
T=\lambda_{0}+k\left(\tilde{\lambda}-\lambda_{0}\right),
$$

where the constant $k, 0 \leq k \leq 1$, is a shrinkage factor specified by the experimenter according to his belief in the guess value. To resolve the uncertainty that $\lambda_{0}$ is approximately $\lambda$ or not, a preliminary test of $H_{0}: \lambda=\lambda_{0}$ against the alternative $H_{1}: \lambda \neq \lambda_{0}$ at some preassigned level of significance may be considered. The idea of such a preliminary test of significance is given in Bancroft (1944).

Pandey and Malik (1988) have obtained some estimators for the inverse dispersion and have found that they perform better in the sense of a smaller mean squared error if the guess value $\lambda_{0}$ is in the vicinity of the true value. The use of the LINEX loss function in estimation procedures has been considered in different contexts by Pandey (1997), Pandey and Srivastava (2001), and Pandey et al. (2004).

In this paper, we propose shrinkage testimators for $\lambda$ when a prior guess $\lambda_{0}$ is available and study it under the LINEX loss function. In Section 2, we propose a class of estimators $S=c \tilde{\lambda}$ and find the value $c_{1}$ for which the risk under the LINEX loss is minimum. An estimate $\hat{T}$ of $T$ is obtained in Section 3 by minimizing the risk of $T$ under the LINEX loss. The risk of $\hat{T}$ is compared with $\hat{S}=c_{1} \tilde{\lambda}$. Shrinkage testimators of $\lambda$ are proposed in Section 4 and their risks are compared with $\hat{S}$.

\section{A Class of Estimator and its Properties}

The proposed class of estimators for $\lambda$ in the inverse Gaussian distribution (1) is

$$
S=c \tilde{\lambda}
$$

The LINEX loss function of $S$, using a Maclaurin expansion of (2), is

$$
L(S)=b \frac{a^{2}}{2}\left[\left(c \frac{n-3}{\lambda v}-1\right)^{2}+\frac{a}{3}\left(c \frac{n-3}{\lambda v}-1\right)^{3}+O\left(a^{2}\right)\right] .
$$

Hence, the risk under the LINEX loss for $S$, ignoring the terms $O\left(a^{2}\right)$ is

$$
R(S)=b \frac{a^{2}}{2}\left[\frac{c^{3} a(n-3)^{2}}{3(n-5)(n-7)}+\frac{c^{2}(1-a)(n-3)}{(n-5)}+c(a-2)+1-\frac{a}{3}\right] .
$$


The value of $c$ for which $R(S)$ is minimum, is

$$
c_{1}=\frac{a-1+\sqrt{1-2 a(a-2) /(n-7)}}{a(n-3) /(n-7)}, \quad n>7 .
$$

Hence, the estimator

$$
\hat{S}=c_{1} \tilde{\lambda}=c_{1} \frac{n-3}{v}
$$

is the estimator of $\lambda$ with minimum risk in the class $S=c \tilde{\lambda}$. The value of $c$ obtained by minimizing the mean squared error of $S=c \tilde{\lambda}$ is

$$
c^{\prime}=\frac{n-5}{n-3}, \quad n>3 .
$$

Thus, the usual estimator $S^{*}=(n-5) / v$ is inadmissible under the LINEX loss and $c_{1} \rightarrow c^{\prime}$ as $a \rightarrow 0$. The risk of the estimator $\hat{S}$ under the LINEX loss is given by

$$
R(\hat{S})=b\left(\exp (-a) I\left(\infty, c_{1}, 1\right)+\left(a-a c_{1}-1\right)\right)
$$

where

$$
I(u, v, r)=\frac{1}{\Gamma\left(\frac{n-1}{2}\right)} \int_{0}^{u} \exp (-z+v a(n-3) / 2 z) z^{(n-1) / 2-r} d z
$$

\section{The Shrinkage Estimator and its Properties}

The risk of the shrinkage estimator $T$ under the LINEX loss as obtained for $S$ is

$$
R(T)=b \frac{a^{2}}{2} \mathrm{E}\left[\left(k^{2} \beta_{1}^{2}+2 \beta_{1} \beta_{2} k+\beta_{2}^{2}\right)+\frac{a}{3}\left(k^{3} \beta_{1}^{3}+\beta_{2}^{3}+3 k^{2} \beta_{1}^{2} \beta_{2}+3 k \beta_{1} \beta_{2}^{2}\right)\right],
$$

where $\beta_{1}=(n-3) / v \lambda-\beta, \beta_{2}=\beta-1$, and $\beta=\lambda_{0} / \lambda$. The value of $k$ minimizing $R(T)$ is

$$
k_{1}=\frac{-B^{\prime}+\sqrt{B^{\prime 2}-4 A^{\prime} C^{\prime}}}{2 A^{\prime}}, \quad B^{\prime 2}-4 A^{\prime} C^{\prime}>0, \quad A^{\prime} \neq 0
$$

where

$$
\begin{aligned}
& A^{\prime}=a\left[\frac{(n-3)^{2}}{(n-5)(n-7)}-\beta^{3}-3 \beta \frac{n-3}{n-5}+3 \beta^{2}\right], \\
& B^{\prime}=2(1+a(\beta-1))\left(\frac{n-3}{n-5}+\beta^{2}-2 \beta\right), \\
& C^{\prime}=-(1-\beta)^{2}(2+a(\beta-1)) .
\end{aligned}
$$

The value $k_{1}$ depends on the unknown parameter $\lambda^{r}, r=1,2,3$. An estimate $k_{2}$ of $k_{1}$ can be obtained by replacing $\lambda^{r}$ by its unbiased estimate

$$
\hat{\lambda}^{r}=\frac{2^{r} \Gamma\left(\frac{n-1}{2}\right)}{v^{r} \Gamma\left(\frac{n-1}{2}-r\right)}, \quad r=1,2,3 .
$$


The corresponding estimate of $T$ is then

$$
\hat{T}=\lambda_{0}+k_{2}\left(\tilde{\lambda}-\lambda_{0}\right) .
$$

The risk of the estimator $\hat{T}$ under the LINEX loss is

$$
R(\hat{T})=b\left[e^{a(\beta-1)} G\left(\infty, k_{2}, 1, f_{2}\right)+a \beta G\left(\infty, 0,1, f_{1}\right)-a \frac{n-3}{2} G\left(\infty, 0,2, f_{1}\right)+a-a \beta-1\right]
$$

where

$$
G\left(u, v, r, f_{i}\right)=\frac{1}{\Gamma\left(\frac{n-1}{2}\right)} \int_{0}^{u} f_{i} \exp (-z+v a(n-3) / 2 z) z^{\frac{n-1}{2}-r} d z
$$

$i=1,2$, and $f_{1}=k_{2}, f_{2}=\exp \left(-a k_{2} \beta\right)$.

The relative efficiency of $\hat{T}$ compared to $\hat{S}$ is defined as

$$
R E(\hat{T}, \hat{S})=\frac{R(\hat{S})}{R(\hat{T})}
$$

which is a function in $n, a$, and $\beta$. For the values $n=10,15,25,35, a=-0.50(0.25) 0.50$ with $a \neq 0$, and $\beta=0.25(0.25) 1.75$, the relative efficiencies are presented in Table 1 . This table shows that the shrinkage estimator $\hat{T}$ has smaller risk than $\hat{S}$ if $\lambda_{0}$ is in the vicinity of $\lambda$. More specifically, if $n$ is small and $0.25 \leq \beta \leq 1.75$, the proposed shrinkage estimator is preferable to the class of unbiased estimators under the LINEX loss.

Table 1: Relative Efficiency $(\tilde{T}, \hat{S})$

\begin{tabular}{rrrcccccc}
\hline & & & & \multicolumn{6}{c}{$\beta$} \\
$n$ & $a$ & 0.25 & 0.50 & 0.75 & 1.00 & 1.25 & 1.50 & 1.75 \\
\hline \multirow{4}{*}{10} & -0.50 & 1.0626 & 1.2683 & 1.6163 & 1.7417 & 1.6648 & 1.5332 & 1.3763 \\
& -0.25 & 1.1184 & 1.4100 & 1.8321 & 1.9274 & 1.7356 & 1.5047 & 1.2973 \\
& 0.25 & 1.2001 & 1.5431 & 1.9544 & 2.0092 & 1.7671 & 1.4849 & 1.2514 \\
& 0.50 & 1.1913 & 1.3478 & 1.4829 & 1.4905 & 1.4044 & 1.2872 & 1.1719 \\
\hline \multirow{4}{*}{15} & -0.50 & 1.0683 & 1.2065 & 1.6784 & 1.9078 & 1.6811 & 1.4170 & 1.2068 \\
& -0.25 & 1.0857 & 1.2488 & 1.7386 & 1.9527 & 1.6881 & 1.3944 & 1.1716 \\
& 0.25 & 1.1194 & 1.3177 & 1.8173 & 1.9933 & 1.6748 & 1.3398 & 1.0987 \\
& 0.50 & 1.1527 & 1.2209 & 1.3310 & 1.3647 & 1.3171 & 1.2435 & 1.1700 \\
\hline \multirow{4}{*}{25} & -0.50 & 1.0821 & 1.0880 & 1.5375 & 1.9508 & 1.6026 & 1.2259 & 1.0615 \\
& -0.25 & 1.0882 & 1.1082 & 1.5661 & 1.9656 & 1.5896 & 1.2031 & 1.0335 \\
& 0.25 & 1.1013 & 1.1458 & 1.6148 & 1.9832 & 1.5578 & 1.1547 & 0.9754 \\
& 0.50 & 1.1195 & 1.1645 & 1.5001 & 1.7182 & 1.4435 & 1.1463 & 0.9968 \\
\hline & -0.50 & 1.0960 & 1.0484 & 1.4347 & 1.9393 & 1.5282 & 1.1283 & 0.9910 \\
35 & -0.25 & 1.0992 & 1.0623 & 1.4555 & 1.9484 & 1.5136 & 1.1071 & 0.9678 \\
& 0.25 & 1.1062 & 1.0888 & 1.4929 & 1.9608 & 1.4815 & 1.0631 & 0.9196 \\
& 0.50 & 1.1101 & 1.1016 & 1.5083 & 1.9595 & 1.4627 & 1.0406 & 0.8953 \\
\hline
\end{tabular}




\section{Shrinkage Testimators and their Properties}

In Section 3 we have seen that $\hat{T}$ has smaller risk than the usual estimator $\hat{S}$ when $\lambda_{0}$ is in the vicinity of $\lambda$. This suggests that on the basis of a given set of data, we should first test $H_{0}: \lambda=\lambda_{0}$. If $H_{0}$ is accepted, $\hat{T}$ is taken as the estimator of $\lambda$, otherwise we use $\hat{S}$ instead of. Thus, we propose a shrinkage testimator for the inverse dispersion as

$$
T^{*}= \begin{cases}k_{2}(n-3) / v+\left(1-k_{2}\right) \lambda_{0}, & \text { if } H_{0}: \lambda=\lambda_{0} \text { is accepted, } \\ c_{1}(n-3) / v, & \text { otherwise. }\end{cases}
$$

Muniruzzaman (1957) obtained a test statistic for testing $H_{0}: \lambda=\lambda_{0}$ against the alternative $H_{1}: \lambda \neq \lambda_{0}$. Under $H_{0}$ we have $\lambda_{0} v \sim \chi_{n-1}^{2}$ and

$$
H_{0} \text { is rejected if } r_{1} \leq \lambda_{0} v \leq r_{2} \text { or if } t_{1} \leq v \leq t_{2},
$$

where $t_{1}=r_{1} / \lambda_{0}, t_{2}=r_{2} / \lambda_{0}$ with $r_{1}$ and $r_{2}$ being the values of the lower and upper $100 \alpha / 2 \%$ points of the chi-squared distribution with $n-1$ degrees of freedom. Thus

$$
T^{*}= \begin{cases}k_{2}(n-3) / v+\left(1-k_{2}\right) \lambda_{0}, & \text { if } t_{1} \leq v \leq t_{2}, \\ c_{1}(n-3) / v, & \text { if } v \leq t_{1} \text { or } v \geq t_{2} .\end{cases}
$$

The risk under the LINEX loss of $T^{*}$ is

$$
\begin{aligned}
R\left(T^{*}\right)= & b\left\{e^{a(\beta-1)}\left[G\left(z_{2}, k_{2}, 1, f_{2}\right)-G\left(z_{1}, k_{2}, 1, f_{2}\right)\right]+e^{-a} I\left(\infty, c_{1}, 1\right)\right. \\
& -e^{-a}\left[I\left(z_{2}, c_{1}, 1\right)-I\left(z_{1}, c_{1}, 1\right)\right]+a \beta\left[G\left(z_{2}, 0,1, f_{1}\right)-G\left(z_{1}, 0,1, f_{1}\right)\right] \\
& -a \frac{n-3}{2}\left[G\left(z_{2}, 0,2, f_{1}\right)-G\left(z_{1}, 0,2, f_{1}\right)\right]-a \beta\left[I\left(z_{2}, 0,1\right)-I\left(z_{1}, 0,1\right)\right] \\
& \left.+a c_{1} \frac{n-3}{2}\left[I\left(z_{2}, 0,2\right)-I\left(z_{1}, 0,2\right)\right]+\left(a-a c_{1}-1\right)\right\}
\end{aligned}
$$

where $I(\cdot)$ and $G(\cdot)$ are defined in (6) and (7), respectively. Also, $z_{1}=r_{1} / 2 \beta$ and $z_{2}=r_{2} / 2 \beta$. The relative risk efficiency is $R E\left(T^{*}, \hat{S}\right)=R(\hat{S}) / R\left(T^{*}\right)$. On the other hand, if $H_{0}$ is accepted then

$$
r_{1} \leq \lambda_{0} v \leq r_{2} \Longleftrightarrow 0 \leq \frac{\lambda_{0} v-r_{1}}{r_{2}-r_{1}} \leq 1
$$

Thus, one choice of the shrinkage factor based on the test statistic is

$$
k_{3}=\frac{\lambda_{0} v-r_{1}}{r_{2}-r_{1}}
$$

With this shrinkage factor, the proposed testimator is

$$
T^{* *}= \begin{cases}k_{3}(n-3) / v+\left(1-k_{3}\right) \lambda_{0}, & \text { if } t_{1} \leq v \leq t_{2}, \\ c_{1}(n-3) / v, & \text { if } v \leq t_{1} \text { or } v \geq t_{2} .\end{cases}
$$

The risk of the testimator $T^{* *}$ can be obtained similarly as for $T^{*}$ by simply replacing $k_{2}$ with $k_{3}$. 
The relative efficiency then is $R E\left(T^{* *}, \hat{S}\right)=R(\hat{S}) / R\left(T^{* *}\right)$. The relative efficiencies $R E\left(T^{*}, \hat{S}\right)$ and $R E\left(T^{* *}, \hat{S}\right)$ are both functions in $n, a, \beta$, and $\alpha$ (the level of significance). For the values $n=10,15,25,35, a=-0.50(0.25) 0.50$ with $a \neq 0, \beta=0.25(0.25) 1.75$, and $\alpha=0.01,0.05$, we have calculated these values and present them in Tables 2 and 3 . From these tables we conclude that the shrinkage testimator $T^{*}$ has smaller risk than $\hat{S}$ when $0.25 \leq \beta \leq 1.50$ for fixed $n, a$, and $\alpha$. On the other hand, the shrinkage testimator $T^{* *}$ is efficient when $0.75 \leq \beta \leq 1.50$ for all considered values of $n$, $a$, and $\alpha$. The efficiencies of $T^{*}$ and $T^{* *}$ are maximum at $\beta=1$, where the gain in efficiency of $T^{* *}$ is larger than $T^{*}$. The effective intervals of $T^{*}$ and $T^{* *}$ decrease as $n$ increases.

Table 2: Relative Efficiency $\left(T^{*}, \hat{S}\right)$

\begin{tabular}{|c|c|c|c|c|c|c|c|c|c|}
\hline$n$ & $a$ & $\alpha$ & 0.25 & 0.50 & 0.75 & 1.00 & 1.25 & 1.50 & 1.75 \\
\hline \multirow{8}{*}{10} & \multirow{2}{*}{-0.50} & 0.01 & 1.3268 & 1.4791 & 1.9142 & 2.1423 & 2.0790 & 1.8968 & 1.7115 \\
\hline & & 0.05 & 1.4357 & 1.4359 & 1.7319 & 1.9250 & 1.8925 & 1.7455 & 1.5889 \\
\hline & \multirow{2}{*}{-0.25} & 0.01 & 1.4435 & 1.7309 & 2.2549 & 2.4193 & 2.1851 & 1.8542 & 1.5728 \\
\hline & & 0.05 & 1.4966 & 1.7292 & 2.1722 & 2.2955 & 2.0417 & 1.7050 & 1.4349 \\
\hline & \multirow{2}{*}{0.25} & 0.01 & 1.5593 & 1.9980 & 2.5277 & 2.5893 & 2.2448 & 1.8390 & 1.5123 \\
\hline & & 0.05 & 1.5393 & 1.9837 & 2.5494 & 2.5987 & 2.1868 & 1.7349 & 1.3998 \\
\hline & \multirow{2}{*}{0.50} & 0.01 & 1.5464 & 1.7648 & 1.9468 & 1.9548 & 1.8304 & 1.6577 & 1.4880 \\
\hline & & 0.05 & 1.5253 & 1.7519 & 1.9595 & 1.9701 & 1.8223 & 1.6215 & 1.4352 \\
\hline \multirow{8}{*}{15} & \multirow{2}{*}{-0.50} & 0.01 & 1.4299 & 1.4584 & 2.0496 & 2.4002 & 2.1160 & 1.7044 & 1.4032 \\
\hline & & 0.05 & 1.4879 & 1.4727 & 1.9252 & 2.2262 & 1.9434 & 1.5512 & 1.2877 \\
\hline & \multirow{2}{*}{-0.25} & 0.01 & 1.4506 & 1.5288 & 2.1367 & 2.4594 & 2.1206 & 1.6745 & 1.3570 \\
\hline & & 0.05 & 1.4931 & 1.5469 & 2.0390 & 2.3067 & 1.9510 & 1.5180 & 1.2376 \\
\hline & \multirow{2}{*}{0.25} & 0.01 & 1.4815 & 1.6489 & 2.2671 & 2.5215 & 2.1026 & 1.6147 & 1.2790 \\
\hline & & 0.05 & 1.4998 & 1.6623 & 2.2197 & 2.4215 & 1.9559 & 1.4677 & 1.1640 \\
\hline & \multirow{2}{*}{0.50} & 0.01 & 1.4978 & 1.5570 & 1.6847 & 1.7180 & 1.6430 & 1.5244 & 1.4083 \\
\hline & & 0.05 & 1.5006 & 1.5587 & 1.6806 & 1.7077 & 1.6167 & 1.4822 & 1.3619 \\
\hline \multirow{8}{*}{25} & \multirow{2}{*}{-0.50} & 0.01 & 1.2927 & 1.3201 & 1.8706 & 2.4585 & 1.9795 & 1.4490 & 1.1702 \\
\hline & & 0.05 & 1.2996 & 1.3862 & 1.7586 & 2.2721 & 1.8009 & 1.3249 & 1.1157 \\
\hline & \multirow{2}{*}{-0.25} & 0.01 & 1.2940 & 1.3533 & 1.9093 & 2.4756 & 1.9641 & 1.4209 & 1.1375 \\
\hline & & 0.05 & 1.3997 & 1.4163 & 1.8086 & 2.2966 & 1.7851 & 1.2950 & 1.0800 \\
\hline & \multirow{2}{*}{0.25} & 0.01 & 1.3961 & 1.4159 & 1.9793 & 2.4961 & 1.9286 & 1.3666 & 1.0763 \\
\hline & & 0.05 & 1.3998 & 1.4691 & 1.9019 & 2.3375 & 1.7550 & 1.2414 & 1.0157 \\
\hline & \multirow{2}{*}{0.50} & 0.01 & 1.4276 & 1.4577 & 1.8638 & 2.1650 & 1.7947 & 1.3733 & 1.1253 \\
\hline & & 0.05 & 1.4899 & 1.4940 & 1.8202 & 2.0795 & 1.6774 & 1.2719 & 1.0706 \\
\hline \multirow{8}{*}{35} & \multirow{2}{*}{-0.50} & 0.01 & 1.1997 & 1.2980 & 1.7183 & 2.4734 & 1.8459 & 1.2939 & 1.0804 \\
\hline & & 0.05 & 1.2200 & 1.3952 & 1.6230 & 2.2817 & 1.6743 & 1.2070 & 1.0798 \\
\hline & \multirow{2}{*}{-0.25} & 0.01 & 1.2998 & 1.3205 & 1.7461 & 2.4825 & 1.8291 & 1.2698 & 1.0531 \\
\hline & & 0.05 & 1.3500 & 1.4119 & 1.6580 & 2.2950 & 1.6568 & 1.1809 & 1.0495 \\
\hline & \multirow{2}{*}{0.25} & 0.01 & 1.4998 & 1.3633 & 1.7988 & 2.4935 & 1.7938 & 1.2231 & 1.0009 \\
\hline & & 0.05 & 1.3525 & 1.4414 & 1.7256 & 2.3179 & 1.6234 & 1.1322 & 0.9919 \\
\hline & \multirow{2}{*}{0.50} & 0.01 & 1.2999 & 1.3840 & 1.8227 & 2.4902 & 1.7738 & 1.2006 & 0.9764 \\
\hline & & 0.05 & 1.3562 & 1.4546 & 1.7575 & 2.3235 & 1.6066 & 1.1099 & 0.9650 \\
\hline
\end{tabular}


Table 3: Relative Efficiency $\left(T^{* *}, \hat{S}\right)$

\begin{tabular}{|c|c|c|c|c|c|c|c|c|c|}
\hline \multirow[b]{2}{*}{$n$} & \multirow[b]{2}{*}{$a$} & \multirow[b]{2}{*}{$\alpha$} & \multicolumn{7}{|c|}{ p } \\
\hline & & & 0.25 & 0.50 & 0.75 & 1.00 & 1.25 & 1.50 & 1.75 \\
\hline \multirow{8}{*}{10} & \multirow{2}{*}{-0.50} & 0.01 & 0.9084 & 1.1066 & 2.3008 & 4.2514 & 4.0342 & 2.6386 & 1.7857 \\
\hline & & 0.05 & 1.3143 & 1.2069 & 1.8041 & 2.5215 & 2.4694 & 1.9617 & 1.5447 \\
\hline & \multirow{2}{*}{-0.25} & 0.01 & 1.0104 & 1.2519 & 2.6105 & 4.7731 & 4.1340 & 2.5036 & 1.6219 \\
\hline & & 0.05 & 1.3831 & 1.4292 & 2.2384 & 3.0491 & 2.6491 & 1.8912 & 1.3948 \\
\hline & \multirow{2}{*}{0.25} & 0.01 & 1.2127 & 1.5194 & 2.7341 & 4.0870 & 3.5184 & 2.3066 & 1.5508 \\
\hline & & 0.05 & 1.4635 & 1.7036 & 2.5777 & 3.2085 & 2.6580 & 1.8756 & 1.3682 \\
\hline & \multirow{2}{*}{0.50} & 0.01 & 1.3725 & 1.5595 & 1.9944 & 2.2534 & 2.1447 & 1.8298 & 1.5109 \\
\hline & & 0.05 & 1.4911 & 1.6404 & 1.9643 & 2.1151 & 1.9648 & 1.6817 & 1.4196 \\
\hline \multirow{8}{*}{15} & \multirow{2}{*}{-0.50} & 0.01 & 1.0737 & 0.8785 & 2.1230 & 4.9376 & 3.5551 & 1.9217 & 1.2645 \\
\hline & & 0.05 & 1.4376 & 1.1048 & 1.8020 & 2.9340 & 2.4123 & 1.6116 & 1.2063 \\
\hline & \multirow{2}{*}{-0.25} & 0.01 & 1.1214 & 0.9347 & 2.1857 & 4.9257 & 3.5360 & 1.8950 & 1.2307 \\
\hline & & 0.05 & 1.4490 & 1.1751 & 1.9016 & 3.0165 & 2.4136 & 1.5807 & 1.1643 \\
\hline & \multirow{2}{*}{0.25} & 0.01 & 1.2062 & 1.0486 & 2.2761 & 4.7069 & 3.4296 & 1.8453 & 1.1782 \\
\hline & & 0.05 & 1.4662 & 1.3021 & 2.0643 & 3.0994 & 2.4007 & 1.5370 & 1.1048 \\
\hline & \multirow{2}{*}{0.50} & 0.01 & 1.4091 & 1.3495 & 1.6835 & 1.8961 & 1.8121 & 1.5864 & 1.3717 \\
\hline & & 0.05 & 1.4376 & 1.1048 & 1.8020 & 2.9340 & 2.4123 & 1.6116 & 1.2063 \\
\hline \multirow{8}{*}{25} & \multirow{2}{*}{-0.50} & 0.01 & 1.4388 & 0.6727 & 1.5854 & 4.9915 & 2.7099 & 1.3909 & 0.9944 \\
\hline & & 0.05 & 1.4978 & 1.0056 & 1.4449 & 2.9701 & 2.0788 & 1.3080 & 1.0532 \\
\hline & \multirow{2}{*}{-0.25} & 0.01 & 1.4456 & 0.7050 & 1.6166 & 4.9905 & 2.7074 & 1.3702 & 0.9663 \\
\hline & & 0.05 & 1.4981 & 1.0436 & 1.4881 & 2.9958 & 2.0680 & 1.2812 & 1.0185 \\
\hline & \multirow{2}{*}{0.25} & 0.01 & 1.4568 & 0.7709 & 1.6757 & 4.9375 & 2.6951 & 1.3314 & 0.9145 \\
\hline & & 0.05 & 1.4986 & 1.1158 & 1.5713 & 3.0317 & 2.0478 & 1.2342 & 0.9565 \\
\hline & \multirow{2}{*}{0.50} & 0.01 & 1.4702 & 0.9015 & 1.6516 & 3.2071 & 2.2686 & 1.3515 & 0.9825 \\
\hline & & 0.05 & 1.4990 & 1.2158 & 1.5843 & 2.4565 & 1.8808 & 1.2690 & 1.0179 \\
\hline \multirow{8}{*}{35} & \multirow{2}{*}{-0.50} & 0.01 & 1.4975 & 0.6492 & 1.2583 & 4.9016 & 2.2313 & 1.1792 & 0.9331 \\
\hline & & 0.05 & 1.5000 & 1.0690 & 1.2237 & 2.9631 & 1.8443 & 1.1832 & 1.0485 \\
\hline & \multirow{2}{*}{-0.25} & 0.01 & 1.4978 & 0.6765 & 1.2820 & 4.9174 & 2.2270 & 1.1587 & 0.9056 \\
\hline & & 0.05 & 1.5000 & 1.0969 & 1.2542 & 2.9812 & 1.8321 & 1.1574 & 1.0158 \\
\hline & \multirow{2}{*}{0.25} & 0.01 & 1.4982 & 0.7315 & 1.3288 & 4.9207 & 2.2164 & 1.1195 & 0.8535 \\
\hline & & 0.05 & 1.5000 & 1.1495 & 1.3148 & 3.0094 & 1.8096 & 1.1099 & 0.9542 \\
\hline & \multirow{2}{*}{0.50} & 0.01 & 1.4984 & 0.7605 & 1.3524 & 4.8718 & 2.2061 & 1.1014 & 0.8296 \\
\hline & & 0.05 & 1.5000 & 1.1750 & 1.3455 & 3.0097 & 1.7975 & 1.0885 & 0.9258 \\
\hline
\end{tabular}

\section{Summary}

A minimum risk estimator under the LINEX loss for the inverse dispersion $\lambda$ of the inverse Gaussian distribution in the class of unbiased estimator has been proposed. As a shrinkage estimator performs better, if the guess value $\lambda_{0}$ is in the vicinity of true value $\lambda$, the class of shrinkage estimators have also been proposed and compared with the minimum risk estimator under the LINEX loss. By choosing the shrinkage factor, two testimators $T^{*}$ and $T^{* *}$ have been obtained. On the basis of numerical finding, we observed that $T^{*}$ performs better if $0.25 \leq \lambda_{0} / \lambda \leq 1.50$ and the estimator $T^{* *}$ performs better if $0.75 \leq \lambda_{0} / \lambda \leq 1.50$. Both these testimators have maximum relative efficiency when $\lambda$ is equal to $\lambda_{0}$. 


\section{Acknowledgments}

The authors are thankful to the referees for their helpful comments and suggestions for clarity and improvement of the paper.

\section{References}

Bancroft, T. A. (1944). On biases in estimation due to the use of preliminary test of significance. The Annals of Mathematical Statistics, 15, 190-204.

Folks, J. L., and Chhikara, R. S. (1978). The inverse gaussian distribution and its statistical application - a review. Journal of the Royal Statistical Society, B, 40, 263-289.

Muniruzzaman, A. N. M. (1957). On measures of location and dispersion and test of hypothesis on a Pareto distribution. Calcutta Statistical Association Bulletin, 7, 115-123.

Pandey, B. N. (1997). Testimator of the scale parameter of the exponential distribution using LINEX loss function. Communications in Statistics - Theory and Methods, 26, 2191-2200.

Pandey, B. N., and Malik, H. J. (1988). Some improved estimators for a measure of dispersion of an inverse gaussian distribution. Communications in Statistics - Theory and Methods, 17, 3935-3949.

Pandey, B. N., and Srivastava, A. K. (2001). Estimation of variance using asymmetric loss function. IAPQR Transactions, 26, 109-123.

Pandey, B. N., Srivastava, A. K., and Mishra, G. C. (2004). Invariant version of LINEX loss function and its applications in exponential type II censored data. Aligarh Journal of Statistics, 24, 1-22.

Seshadri, V. (1998). The Inverse Gaussian Distribution: Statistical Theory and Applications. New York: Springer Verlag.

Thompson, J. R. (1968). Some shrinkage techniques for estimating the mean. Journal of the American Statistical Association, 63, 113-122.

Tweedie, M. C. K. (1957a). Statistical properties of inverse gaussian distribution - I. The Annals of Mathematical Statistic, 28, 362-377.

Tweedie, M. C. K. (1957b). Statistical properties of inverse gaussian distribution - II. The Annals of Mathematical Statistic, 28, 696-705.

Varian, H. R. (1975). A bayesian approach to real estate assessment. In S. E. Feinberge and A. Zellner (Eds.), Studies in Bayesian Econometrics and Statistics in honor of L.J. Savage (p. 195-208). Amsterdam: North Holland.

Zellner, A. (1986). Bayesian estimation and prediction using asymmetric loss function. Journal of the American Statistical Association, 81, 446-451.

Authors' address:

Gyan Prakash and D. C. Singh

Department of Statistics,

Harishchandra P. G. College

Varanasi, Uttar Pradesh, India

E-mail: ggyanji@yahoo.com 